\title{
Apaf1 reduced expression levels generate a mutant phenotype in adult brain and skeleton
}

\author{
DOI: $10.1038 / \mathrm{sj} / \mathrm{cdd} / 4400994$
}

\section{Dear Editor,}

Apoptosis plays a major role in the morphogenesis and homeostasis of the developing central nervous system (CNS). The apoptosome, activated during development and in the adult brain, is the dynamic effector of the mitochondrial pathway of cell death. The adapter molecule Apaf1 (apoptotic protease activating factor 1) is the core of the apoptosome. ${ }^{1-3}$ Pro-apoptotic molecules such as Bax and Bid and anti-apoptotic molecules such as Bcl- $X_{L}$ or $\mathrm{Bcl}-2$ regulate, upon specific stimuli, the release of cytochrome $c$ from mitochondria. Cytochrome $c$ has been shown to activate the Apaf1 protein, which in turn activates the protease caspase-9 (Casp9), resulting then in cell death. In fact, the knockout of murine Apaf1 leads to embryonic lethality with a phenotype affecting several aspects of developmental apoptosis and particularly brain morphogenesis and histogenesis. ${ }^{4-6}$ The Apaf1 locus maps to chromosome 12q22-q23 in humans and to chromosome 10C3-D1 in mouse. ${ }^{4}$

In 1997, Harris and collaborators reported that Forebrain overgrowth (fog) is an autosomal recessive mutation affecting neural tube development. ${ }^{7}$ This mutation was shown to map in the Apaf1 chromosomal region but the corresponding gene was uncharacterised. The resulting phenotype included mono- or bi-lateral brain bumps with subcutaneous haemorrhage covered by hair-bearing skin in adults. In addition, lumbo-sacral defects (spina bifida) were present in alternative or in association with the phenotype. Adult homozygous mouse tails often showed distal kinks or a corkscrewlike shape, with the first loop at their very base. A certain percentage of the mutants died during gestation around embryonic day 16.5 (e16.5). ${ }^{7}$ Recently, Honarpour et al. ${ }^{8}$ reported that: (a) mutant fog newborn are functionally deficient in Apaf1 protein; (b) Apaf1-transcripts are aberrantly processed in homozygous fog mutants, resulting in reduced levels of the normal Apaf1 message; and (c) Apaf1/fog double heterozygous (DH) embryos show defects consistent with those reported for fog and Apaf1 single homozygotes.

We independently generated Apaf1/fogDH adult mice and characterised their phenotype, which closely resembles the fog/fog phenotype previously described (Figure 1G). Due to the remarkable homogeneity of the genetic background (C57BL/6J), obtained by a number of backcrosses in our laboratory, we suggest that in fact fog represents a hypomorphic allele of Apaf1. This finding offers an interesting model to study the effects of reduction of apoptosome functionally in adult brains with a possible link to human diseases.
Concerning Apaf1/fogDH brain gross anatomy, the most striking feature was the presence of a mono- or bilateral extracranial bump (Figure 1D). This structure appeared to be connected to the right cerebral hemisphere and highly vascularized. Moreover, an unequal development of the two hemispheres was observed, one showing larger size than the other.

Histological examination of mutant mouse telencephalon confirmed the occurrence of cerebral malformations, mainly consisting in an enlargement of one hemisphere, due to the presence of an abnormally high cell number (Figure 1A). Enhanced eosinophylic properties of the altered hemisphere, with respect to the contro-lateral one, were also observed (Figure 1A). Furthermore, the pathological augmentation of the cerebral mass in the rostral part of the neocortex seemed to affect spatial relationships with the ipsi-lateral olfactory bulb, which in turn appears decreased in volume (not shown).

The peculiar forebrain bump showed heterogeneous composition, as it not only contained cerebral tissue, but also loose connective tissue. Numerous blood vessels were seen to connect the bump to the correspondent cerebral hemisphere (Figure 1B, arrow). The same hemisphere also showed one or more highly vascularized infoldings. In these areas, the neocortical cytoarchitecture was profoundly altered, as neuronal layers were hardly recognised. By contrast, the contra-lateral hemisphere showed proper neuronal arrangement in the different cortical layers (Figure 1A).

In the hemisphere bearing the bump and infoldings, several ruptured blood vessels, causing massive intraparenchymal haemorrhages and haemoventricle, were observed (Figure 1A, white arrow). As obvious consequence of haemorrhages, is extensive extra- and intracellular edema (Figure 1C). As we failed to detect any mitotic cells in our adult specimen, we suggest that the observed abnormal enlargement of one cerebral hemisphere results from a defect in the regulation of nervous tissue homeostasis occurred early in ontogenesis.

We also analyzed the gross anatomical malformations at the basis of the kinked tail phenotype. The intervertebral disc takes contact with the ventral side of the caudal vertebra, instead of with its caudal side, generating a sudden bending of the columna (Figure 1E). How an apoptosis deficiency could account for this kind of skeletal abnormality is still unclear. The absence of striking skeletal alteration in the anterior part of the affected caudal vertebra, which retains the proper vertebral processes, 

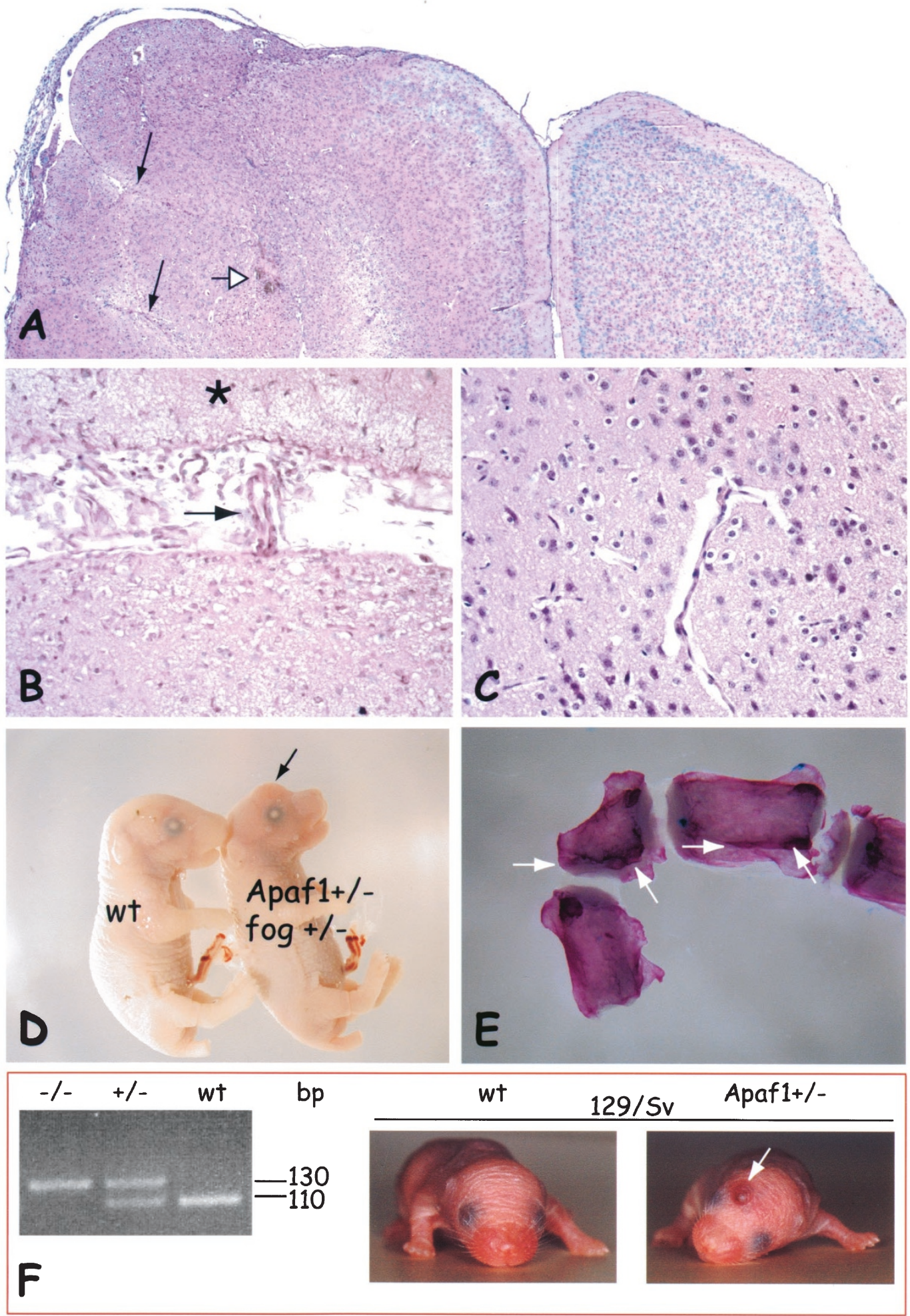

\begin{tabular}{|c|c|c|c|c|c|c|c|c|c|}
\hline & \multirow[t]{2}{*}{ Age (Days) } & \multirow[t]{2}{*}{ No.of litters } & \multirow[t]{2}{*}{$\begin{array}{l}\text { Total } \\
\text { conceptuses }\end{array}$} & \multicolumn{2}{|l|}{$\begin{array}{l}\text { Normal-looking } \\
\text { individuals }\end{array}$} & \multicolumn{3}{|c|}{ Genotype } & \multirow[t]{2}{*}{$\begin{array}{l}\text { Abnormal } \\
\text { individuals }\end{array}$} \\
\hline & & & & & $w t$ & $\mathrm{DH}$ & $A+1-$ & $f+l-$ & \\
\hline & e12-13 & 1 & 9 & 8 & 1 & 1 & 4 & 3 & $1(1)$ \\
\hline & e14-15 & 1 & 10 & 7 & 0 & 3 & 2 & 5 & $3(2)$ \\
\hline & e17-18 & 2 & 15 & 11 & 3 & 4 & 5 & 3 & $4(2)$ \\
\hline & pn30 & 4 & - & 11 & 2 & 3 & 4 & 5 & $3(0)$ \\
\hline
\end{tabular}

(see overleaf for legend) 
seems to suggest the presence of a physiological ossification process.

The anatomical and histological alterations here reported for Apaf1/fogDH mutants are strikingly similar to those described in fog/fog mutants. ${ }^{7}$ Common findings in the two models include the presence of forehead bumps, the nonharmonic development of the two cerebral hemispheres, the massive haemorrhage phenomena, and the presence of a kinky tail. This resemblance strongly supports the hypothesis that indeed fog is an Apaf1 hypomorphic allele, as suggested by the genetic analysis performed by Honarpour and colleagues. ${ }^{8}$ Moreover, the D10Mit230 genetic marker is distinguishable in size (130 bp or $110 \mathrm{bp}$, respectively) between the 129/Sv genetic background (ES cell clone carrying the Apaf1 mutation) and the C57BL/6J genetic background (host), when the Apaf1 mutant embryos and their littermates are analyzed for genotype (Figure 1F, left panel and $\mathrm{G}$ ). This evidence argues for a close link of D10Mit230 with the Apaf1 locus.

In our specific genetic background (F10 in C57BL/6J), the reduced levels of the correct Apaf1 mRNA transcripts correspond to a reduced level of the Apaf1 protein, which guarantees a certain level of apoptosome functionality in the Apaf1/fogDH mice (not shown and ${ }^{8}$ ). Backcrossing our Apaf1 heterozygotes into an inbred background (129/Sv), we observed the occurrence of an Apaf1/fogDH-like or a fog/fog-like phenotype in Apaf $1^{+/}$newborn, implying a striking involvement of Apaf1 dosage in brain morphogenetic and histogenetic cell death, dependent on the genetic background (Figure 1F, right panel).

The interesting phenotype observed raises the possibility that a down-regulation of the Apaf1 gene results in the survival of progenitor cells, which cannot properly arrange to form a normal tissue.

\section{Acknowledgements}

We are indebted to Rainer Libal (Göttingen) for excellent technical assistance, to Valentina Imbroglini (Rome), for microscopic analysis and to Daniela De Zio (Rome) and Joachim Berger (Göttingen) for Apaf1 protein detection in embryonic extracts. We thank Cinzia Bernardi (Azienda Ospedaliera S. Filippo Neri, Roma), for useful discussion. We are grateful to Peter Gruss (Göttingen) for the continuous support and encouragement. F Cecconi is an Assistant Telethon Scientist (Grant Telethon $38 / \mathrm{cp}$ ) and is also supported by AIRC.

\section{$S$ Moreno $^{1}$, E Ferraro $^{2}, S$ Eckert $^{3}$ and F Cecconi ${ }^{\star 2}, 2$}

1Dipartimento di Biologia, Universita' Roma Tre, Roma 00146, Italy;

'Dipartimento di Biologia, Universita' Tor Vergata, Roma 00133, Italy;

${ }^{3}$ Department of Molecular Cell Biology, Max-Planck-Institute of

Biophysical Chemistry, Göttingen, Germany

*Corresponding author: F Cecconi, Dipartimento di Biologia, Universita' Tor Vergata, via della Ricerca scientifica, 00133 Roma, Italy.

Tel: (+39)-06-72594319; Fax: (+39)-06-2023500;

E-mail: francesco.cecconi@uniroma2.it

1. Zou H et al. (1997) Cell $90: 405-413$

2. Li P et al. (1997) Cell 91: 479-489

3. Cecconi F (1999) Cell Death Differ. 6: 1087-1098

4. Cecconi $\mathrm{F}$ et al. (1998) Cell 94: 727-737

5. Yoshida $\mathrm{H}$ et al. (1998) Cell 94: $739-750$

6. Honarpour $\mathrm{N}$ et al. (2000) Dev. Biol. 218: 248-258

7. Harris BS et al. (1997) Teratology 55: 231-240

8. Honarpour N et al. (2001) Proc. Natl. Acad. Sci. USA 98: 9683-9687

9. Kochhar DM (1973) Teratology 7: 289-298

Figure 1 (A) Coronal sections of Apaf1/fogDH mouse telencephalon. PFA fixation, paraffin embedding, H\&E staining. The two hemispheres show evident differences in their size, cytoarchitectural features, and staining properties. Two infoldings (black arrows), preventing cortical layer identification, and a wide haemorrhaged area (white arrow) are visible in the right hemisphere, which shows relatively high eosin affinity. The contra-lateral part is apparently normally structured. (B) Higher magnification of the region connecting the left hemisphere with the extracranial bump (asterisk), showing rich vascularization (arrow). (C) Oedematous area surrounds a blood vessel in the middle of the optic field, showing numerous swollen neurons. (D) A visible extracranial bump in the Apaf1/fogDH e17 embryos, when compared with a wildtype littermate (arrow). (E) The vertebral phenotype in the Apaf1/fogDH adult, at the basis of its kinked tail (post-natal day 30, pn 30). Skeleton preparation was obtained by the $\mathrm{KOH}$ method. ${ }^{9}$ The white arrowheads point to the borders of the ventral vertebral side connected with the following vertebral articulation side (20th caudal). The white arrowheads in the more anterior vertebral body (right) are pointed, for comparison, to the same borders in a normal vertebra (19th caudal). (F) Left panel: genotype of Apaf1 littermates by PCR with primers specific for the D10Mit230 genetic marker. Right panel: the Apaf1 mutant allele is dominant in the inbred genetic background $129 / \mathrm{Sv}$. The white arrow points to the mono-lateral extracranial bump, highly haemorrhaged, in the Apaf1 heterozygous pn0 individual, strikingly similar to the Apaf1/fogDH phenotype. (G) Occurrence of a phenotype by Apaf1/fog breeding. All the embryos and adult individuals with an $A p a f 1 / f o g \mathrm{DH}$ genotype exhibited a phenotype. The numbers into brackets correspond to the individuals with exencephaly and/or spina bifida, persistence of interdigital webs, eye abnormalities (Apaf1 ${ }^{-\prime-}$-like lethal phenotype). A, Apaf1; f, fog; e, embryonic day; pn, post-natal day. Genotypes were obtained as previously described ${ }^{4,8}$ 